\title{
Subjective comparison of brightness preservation methods for local backlight dimming displays
}

\author{
Korhonen, Jari; Mantel, Claire; Forchhammer, Søren
}

\section{Published in:}

Proceedings of the SPIE

Link to article, DOI:

$10.1117 / 12.2079241$

Publication date:

2015

Document Version

Publisher's PDF, also known as Version of record

Link back to DTU Orbit

Citation (APA):

Korhonen, J., Mantel, C., \& Forchhammer, S. (2015). Subjective comparison of brightness preservation methods for local backlight dimming displays. In Proceedings of the SPIE (Vol. 9395, pp. 939504). [939504] SPIE International Society for Optical Engineering. Proceedings of SPIE - The International Society for Optical Engineering https://doi.org/10.1117/12.2079241

\section{General rights}

Copyright and moral rights for the publications made accessible in the public portal are retained by the authors and/or other copyright owners and it is a condition of accessing publications that users recognise and abide by the legal requirements associated with these rights.

- Users may download and print one copy of any publication from the public portal for the purpose of private study or research.

- You may not further distribute the material or use it for any profit-making activity or commercial gain

- You may freely distribute the URL identifying the publication in the public portal 


\title{
Subjective comparison of brightness preservation methods for local backlight dimming displays
}

\author{
J. Korhonen, C. Mantel, S. Forchhammer \\ Technical University of Denmark, Dept. of Photonics Engineering, 2800 Kgs. Lyngby, Denmark
}

\begin{abstract}
Local backlight dimming is a popular technology in high quality Liquid Crystal Displays (LCDs). In those displays, the backlight is composed of contributions from several individually adjustable backlight segments, set at different backlight luminance levels in different parts of the screen, according to the luma of the target image displayed on LCD. Typically, transmittance of the liquid crystal cells (pixels) located in the regions with dimmed backlight is increased in order to preserve their relative brightness with respect to the pixels located in the regions with bright backlight. There are different methods for brightness preservation for local backlight dimming displays, producing images with different visual characteristics. In this study, we have implemented, analyzed and evaluated several different approaches for brightness preservation, and conducted a subjective study based on rank ordering to compare the relevant methods on a real-life LCD with a local backlight dimming capability. In general, our results show that locally adapted brightness preservation methods produce more preferred visual outcome than global methods, but dependency on the content is also observed. Based on the results, guidelines for selecting the perceptually preferred brightness preservation method for local backlight dimming displays are outlined.
\end{abstract}

Keywords: Displays, Local backlight dimming, Image processing, Image quality, Visual effects

\section{INTRODUCTION}

Local backlight dimming is rapidly becoming a popular technique in high quality LCD screens for professional as well as home use. The basic idea of local backlight dimming is simple: the screen is divided in several segments, each with an individually adjustable backlight element. Different backlight intensities can be chosen for each segment, following the brightness of the corresponding regions in the input image. For the television industry, the benefits of local backlight dimming are twofold: first, it improves contrast, as the practical liquid crystal cells cannot block the light perfectly and therefore the light leaking through dark pixels can be reduced by reducing the backlight intensity; and second, it saves energy, since full backlight intensity is not needed for segments with dark content.

In practical local backlight dimming displays, the resolution of the backlight segments is significantly lower than the display resolution. In addition, backlight segments are not optically isolated from each other, and this is why the light from each backlight element is mixed on the passive diffuser plate located between backlight elements and pixels of liquid crystals. Therefore, each pixel receives light from several backlight segments. In many typical real-life images there may be bright and dark pixels located close to each other, and since the backlight intensity tends to be very smooth, a compromise between light leakage in dark pixels and reduced intensity of bright pixels (clipping) is often needed. This makes local backlight dimming a very challenging optimization problem [1-2].

To date, scientific research in local backlight dimming has mainly focused on algorithms determining the backlight levels [1-3]. Some of those algorithms have been evaluated via subjective comparisons, in addition to objective measures of distortion [4], but we are not aware of any prior subjective quality assessment studies comparing techniques for brightness preservation (in some sources, the term "pixel compensation" is used instead) in local backlight dimming displays. Different methods for brightness preservation have been presented in the related work [5-7], but the most sophisticated methods are designed for global backlight dimming, and they are not appropriate in the context of local backlight dimming. Our study is more closely related to studies concerning subjective evaluation of tone mapping

*jark@fotonik.dtu.dk; phone +45 45256594; fax +45 4593 6581; www.fotonik.dtu.dk

Color Imaging XX: Displaying, Processing, Hardcopy, and Applications, edited by Reiner Eschbach, Gabriel G. Marcu, Alessandro Rizzi, Proc. of SPIE-IS\&T Electronic Imaging, SPIE Vol. 9395, 939504 • (c) 2015 SPIE-IS\&T

CCC code: $0277-786 \mathrm{X} / 15 / \$ 18 \cdot$ doi: $10.1117 / 12.2079241$ 
algorithms [8-10]. However, the problem definition for brightness preservation with local backlight dimming is clearly different from classical tone mapping, since conventional tone mapping algorithms do not consider the local variations in the dynamic range of the output device, caused by non-uniform backlight intensity.

In this paper, subjective results comparing several different brightness preservation schemes are presented. The rest of the paper is organized as follows. In Section 2, we introduce the basic concepts of local backlight dimming, and explain brightness preservation in more details. In Section 3, the subjective quality assessment study is described and the results are discussed. Finally, concluding remarks are given in Section 4.

\section{LOCAL BACKLIGHT DIMMING}

A typical local backlight dimming algorithm gets the original digital image as input, and produces backlight intensities and compensated image as output. A block diagram is depicted in Fig. 1. On the diffuser plate, light from each of the backlight elements follows Point Spread Function (PSF) with characteristics depending on the optical properties of the backlight elements and the diffuser. We can assume that changing the backlight intensity changes the amplitude of PSF,

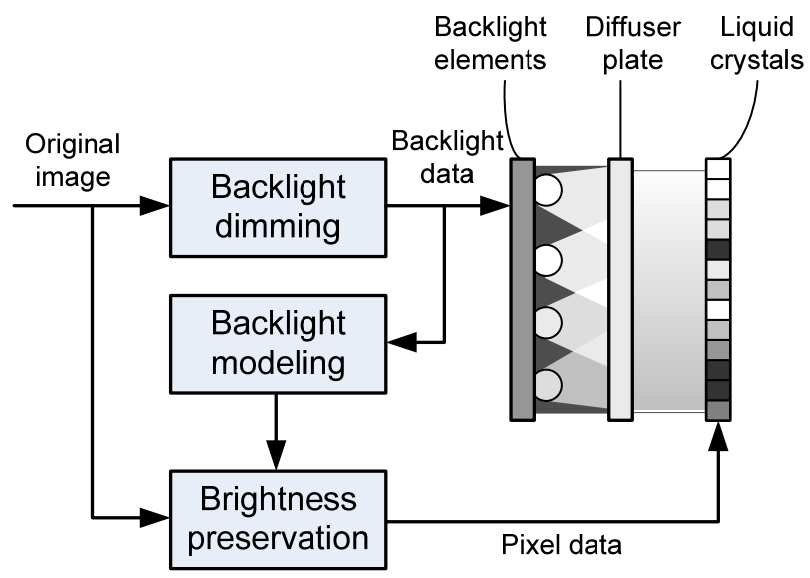

Figure 1. A local backlight dimming display illustrated: backlight dimming algorithm adjusts the intensities of the backlight elements, and brightness preservation modifies original image to fit the backlights.

but not its shape. Then, the backlight intensity on the diffuser plate at each pixel position can be modeled simply by adding the contributions from each backlight element [11]. After this stage, the backlight model can be used to compensate the image according to the chosen brightness preservation strategy.

For the sake of simplicity, in the rest of the paper we assume that backlight intensity $b$ and transmittance $t$ are both normalized to the interval from 0 to 1 , where 1 represents the highest luminance and transmittance levels that can be reached. In addition, we assume that the normalized physical luminance of backlight has been converted to a perceptually uniform scale used also for the pixel data steering the liquid crystal cells. With these assumptions, we can derive the observed pixel luminance $l$ as a product of $b$ and $t$ :

$$
l=b t
$$

Since our focus in this study is not in backlight dimming but brightness preservation and clipping artifacts, we do not present the full backlight model that also includes the impact of light leakage; however, it should be noted that practical backlight dimming algorithms need to trade-off between leakage and clipping. Readers interested in more comprehensive backlight models may refer to the related work in [11-12].

Several backlight dimming algorithms have been proposed to determine the backlight level for each segment. There are algorithms aiming at clipping free image presentation [1], but most algorithms allow some clipping in order to find a trade-off between clipping and leakage artifacts $[2,12]$. Backlight dimming algorithms can be categorized to feature 
based and optimization based algorithms. Feature based algorithms use statistical features of the input image, such as average brightness or histogram of each segment, to compute the backlight intensities [3]. Optimization based algorithms aim at minimizing the average distortion in the backlight model $[1-2,12]$. Lower power consumption with respect to the resulting image quality can be achieved by optimization-based algorithms, but at the cost of higher computational complexity. In this paper, we have used backlight optimization based on Gradient Descent (GD). A power weighting factor can be used to achieve different trade-offs between power consumption and image quality. For the details of the algorithm, reader may refer to [12].

The most straightforward method to perform brightness preservation is hard clipping. Assuming that the modeled backlight intensity at a certain position is $b(b<1)$, and the target pixel brightness is $l_{\text {target }}$, hard clipping computes compensated transmittance $t$ as:

$$
t=\min \left(1, l_{\text {target }} / b\right)
$$

Hard clipping always minimizes the difference between observed brightness $l$ and target brightness $l_{\text {target. }}$ However, as a harmful effect, it causes saturation of bright textures, making fine details in bright areas disappear. If hard clipping is performed separately for the red, green and blue subpixels of a color image, the original hue is not necessarily preserved, since the relative differences between color components may change. For example, orange color may turn into dark yellow, since the red component with high value might be clipped, whereas the green component with lower value may remain below the clipping threshold. The original hue can be preserved by finding the most aggressively clipped color

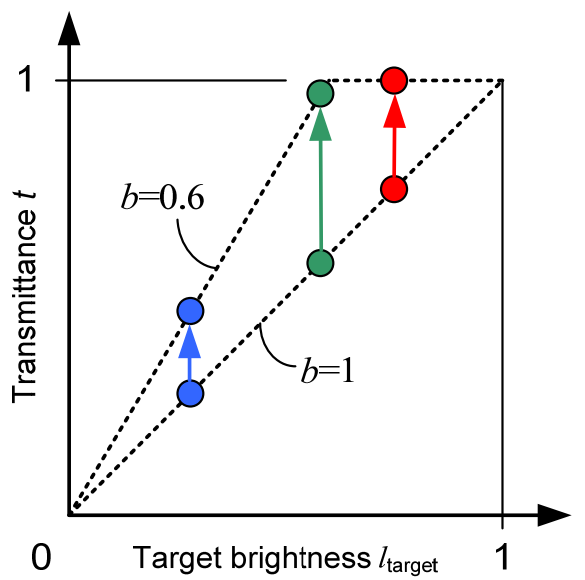

a) Direct hard clipping

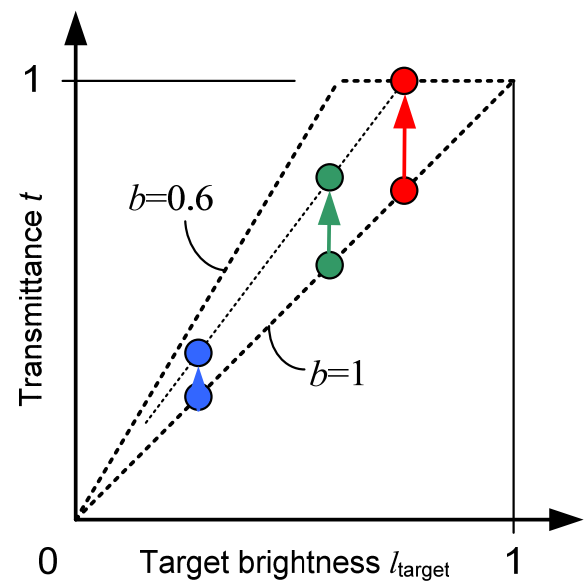

b) Hard clipping with hue preservation

Figure 2. Hard clipping: a) direct clipping of R, G, and B components separately, b) hard clipping with hue preservation.

component and then adjusting the other color components in the respective proportion. Comparison of hard clipping without hue preservation (a) and with hue preservation (b) is shown in Fig. 2.

In order to alleviate the saturation effect in bright areas, it is possible to smoothen the knee point of the clipping curve. This technique is commonly referred to as soft clipping. There are several different ways to implement soft clipping; typically, dark pixels are compensated directly as in hard clipping, whereas bright pixels above a certain rolloff point are compensated according to a nonlinear clipping curve [7]. An example of such soft clipping is shown in Fig. 3 (soft A). In this paper, we have used the soft clipping method described in [13]. This method enhances pixels more conservatively than the more conventional soft clipping schemes relying on relatively large roll-off points, as illustrated in Fig. 3 (soft B). Transmittance is computed as:

$$
t=\left(l_{\text {target }}-(1-b) \cdot l_{\text {target }}^{1 /(1-b)}\right) / b
$$




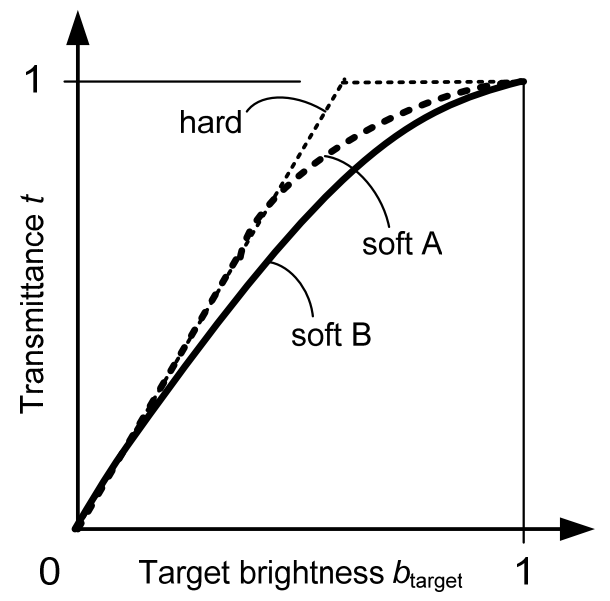

Figure 3. Hard clipping and two types of soft clipping compared $(b=0.6)$.

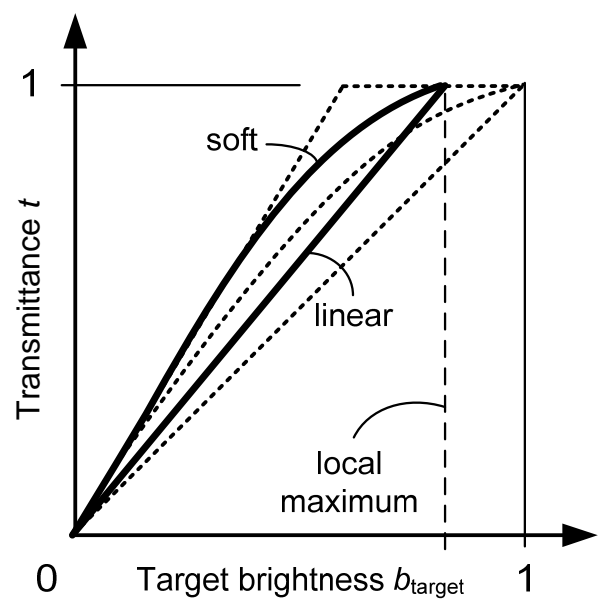

Figure 4. Clipping with respect to local maximum target brightness. Thin dashed lines show clipping in respect to full target brightness (hard, soft and no clipping), thick solid lines show clipping in respect to local maximum (soft and linear).

Since the clipping curve in soft clipping is partially or entirely nonlinear, soft clipping may cause hue distortion if it is applied directly to $\mathrm{R}, \mathrm{G}$ and $\mathrm{B}$ components. This can be avoided by applying hue preservation in a similar manner as in the case of hard clipping. In general, soft clipping produces darker image than hard clipping, but it preserves the details in bright regions significantly better. Visual comparison of different clipping strategies is presented in Fig. 5 .

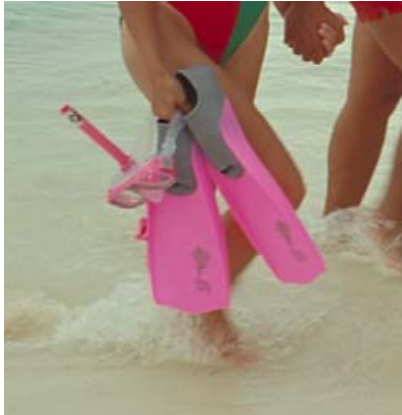

a) Original (full backlight)

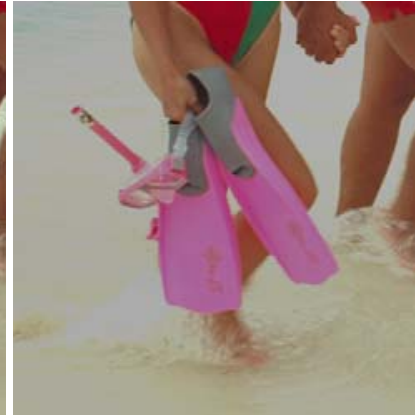

b) Hard clipping

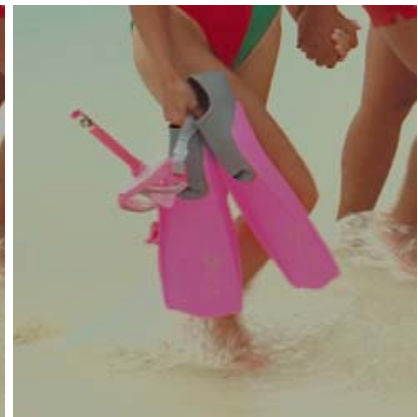

c) Hard + hue preservation

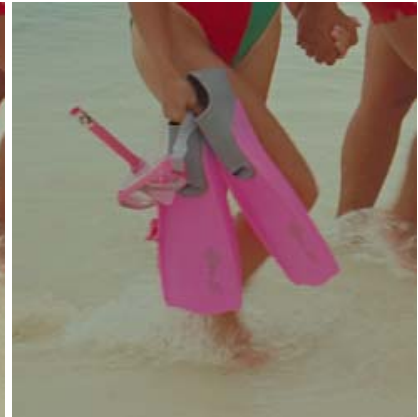

d) Soft clipping

Figure 5. Comparison of different clipping strategies: a) original image, b) hard clipping without hue preservation, c) hard clipping with hue preservation, d) soft clipping.

Earlier in this Section, we have assumed that brightness preservation is performed with respect to the global maximum, i.e. normalized target brightness of 1 . In images with large differences in overall brightness between different regions, this approach does not necessarily give the visually best results. Figure 4 shows how brightness preservation can be performed in case the local maximum of target brightness is known. Two methods are included in this example: linear brightness preservation maps target brightness values from zero to the local maximum linearly into range from 0 to 1 , and soft clipping that also reaches maximum transmittance at the local maximum target brightness. If the local maximum is equal or lower than the backlight, both clipping curves converge towards conventional hard clipping curve. In this case, clipping does not take place, since all the processed pixels have values equal or lower than the local maximum. 
Given local maximum target brightness $l_{\text {localmax }}$, linear enhancement can be formulated as:

$$
t=l_{\text {target }} / l_{\text {localmax }}
$$

If soft clipping is used, an equation for transmittance can be obtained by modifying (3) as follows:

$$
t=\frac{1}{b}\left(l_{\text {target }}-\left(l_{\text {localmax }}-b\right) \cdot\left(\frac{l_{\text {target }}}{l_{\text {localmax }}}\right)^{\frac{l_{\text {localmax }}}{l_{\text {localmax }}-b}}\right)
$$

For the sake of simplicity, it is assumed that in (4) and (5), $l_{\text {localmax }} \geq l_{\text {target. }}$. If it is not the case (for example, if $l_{\text {localmax }}$ is determined suboptimally), full transmittance $(t=1)$ will be used.

There is no standard method to define the local maximum. It should be noted that local maximum should not change drastically between nearby pixels, since it would create undesired blocking or edging effects when different clipping curves were applied to neighboring pixels. For this study, we have used a method where the input image is divided into overlapping blocks, then the maximum pixel value of each block is put into a matrix, and finally the resulting matrix is resized to the same size as the original image, using standard bicubic interpolation. In this way, a map of local maximum values with a smooth gradient is obtained. Overlapping helps to avoid high local variations caused by individual bright pixels located close to block boundaries. In our practical implementation for Full HD resolution (1920x1080 pixels) images, we use roughly square blocks, each made of 192x216 pixels, making 10x5 blocks per image. The workflow for defining the local maxima is illustrated in Fig. 6. More sophisticated methods, based on e.g. image segmentation, could potentially give better results; however, the computational complexity should be reasonable in order to enable real-time implementation.
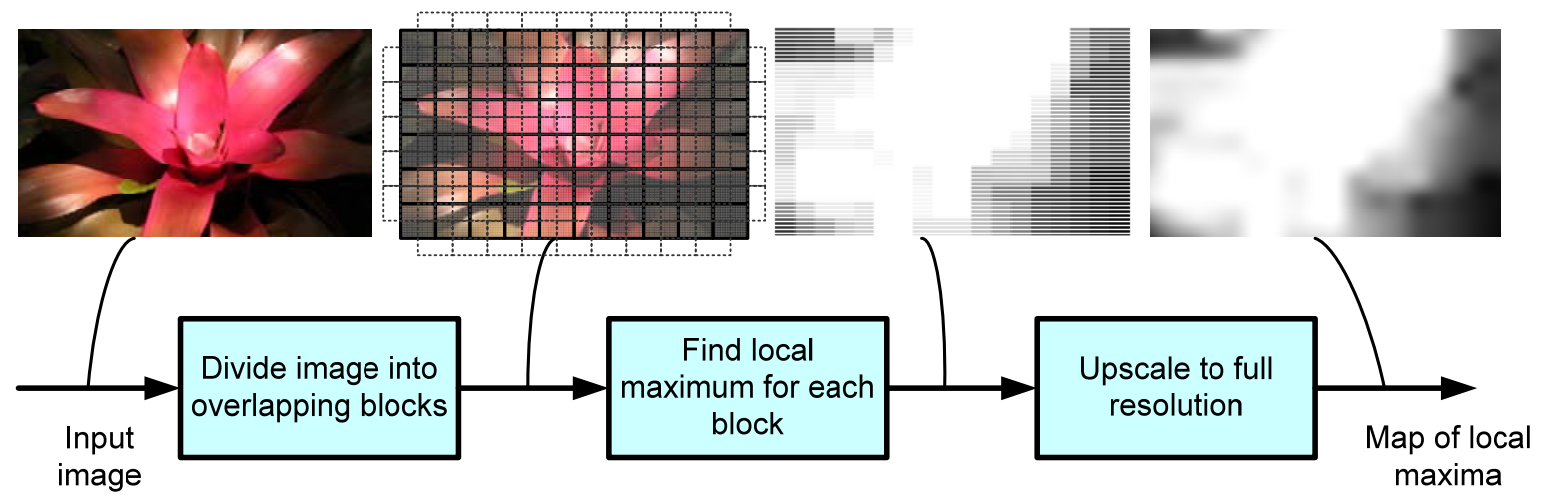

Figure 6. Method for defining local maxima at each pixel position illustrated.

Brightness preservation towards local maximum exploits the dynamic range of the display more efficiently in the dark regions of the image; the relative uniformity of pixels close to each other can be preserved more accurately, saturation is reduced and pixels will still be brighter than if the respective clipping scheme was applied towards global maximum. On the other hand, the disadvantage of this approach is that the relative uniformity between different regions of the image is not preserved. In other words, two pixels with the same target brightness will not appear the same, if they are located in regions with different local maxima.

\section{SUBJECTIVE QUALITY EVALUATION}

To the best of our knowledge, systematic subjective studies comparing the performance of different brightness preservation methods have not been published in the literature. Indeed, reported subjective experiments concerning local backlight dimming displays are scarce in general. The standardized methodologies for subjective quality testing are 
mostly designed for evaluating compression and transmission artifacts [14], and therefore they are not appropriate for experimenting backlight dimming or brightness preservation algorithms. This is why we have developed our own methodology for this study.

\subsection{Methodology and test arrangements}

Since the visible variations between different brightness preservation schemes are often subtle, we have assumed that differences may be difficult to quantify via conventional methods based on numerical ratings. Pairwise comparisons could provide more accurate results, but the problem is the large amount of pairs that need to be tested. This is why we have chosen a rank ordering method, where test subjects sort different versions of an image into an order of preference.

In our methodology, test subjects use a mouse and a simple graphical user interface to switch between different versions of the image, and rearrange them into the order of preference. User interface (UI) is displayed on a small separate display with low luminance, located between the backlight dimming display and test subject. We have chosen the location of the UI display so that a test subject can change focus from test image and user interface with as small eye movements as possible. Figure 7 shows a photo of the test environment.

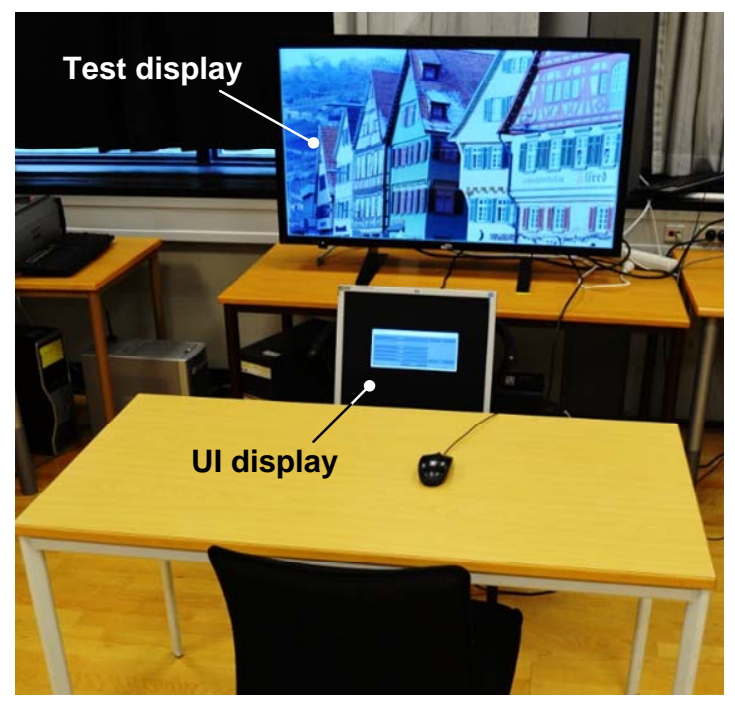

Figure 7. Subjective test environment with the test display showing the test images, and UI display used to order different versions of the images into a preferred rank order.

In our experiment, we have used six images: two different images (Beach and Parrot) from the Kodak database [15], two images (Exotic Flower and Lizard) from the database described in [16], and finally, two images extracted from video sequences LA Diver and LA Volcano from the Consumer Digital Video Library (CDVL) [17]. The original test images are shown in Fig. 8. Two additional images were used for training, not shown in the figure.

All the images were displayed in Full HD resolution, and they have been chosen to represent different overall brightness levels, contrast and colorfulness. Backlight dimming based on gradient descent and power weighting [12] has been applied to each image to optimize the backlight values with respect to estimated Mean Squared Error (MSE). Higher weight on power results in lower power consumption, but more apparent leaking artifacts. We have used relatively high power weights, since we assume that at high power the differences between brightness preservation algorithms would be very difficult to see. Details of the backlight dimming algorithm are omitted in this paper, due to space constraints.

With the given backlight computed by the algorithm from [12], six different versions of each image were created by applying different brightness preservation algorithms: a) hard clipping (Eq. 2) without hue preservation, b) hard clipping with hue preservation c) soft clipping against local maximum (Eq. 5), d) global soft clipping (Eq. 3), e) linear pixel enhancement against local maximum (Eq. 4), and f) no compensation (the original image is shown as such on locally 
dimmed backlight). The order of methods from a) to f) reflects approximately the overall brightness of the resulting image, from brightest to darkest. Characteristics of different methods are summarized in Table 1. As an example, the results from each method when applied to Beach image are shown in Fig. 9.

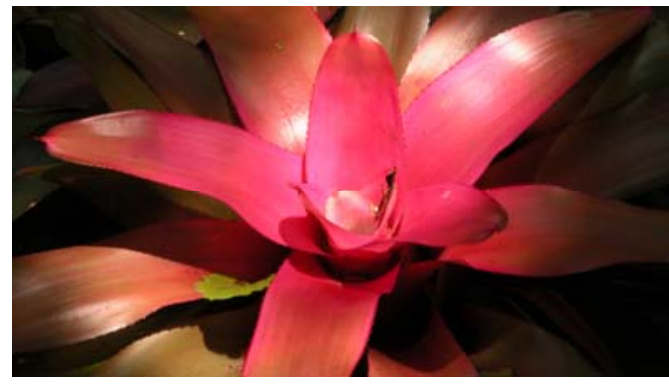

a) Exotic Flower

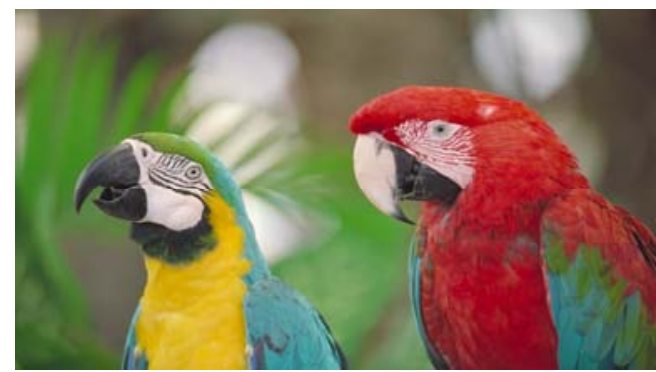

c) Parrot

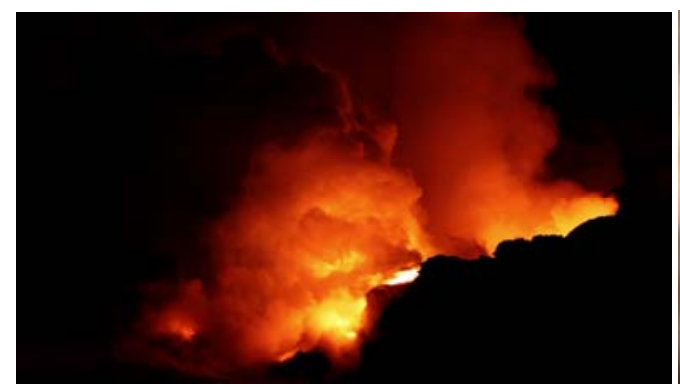

e) LA Volcano

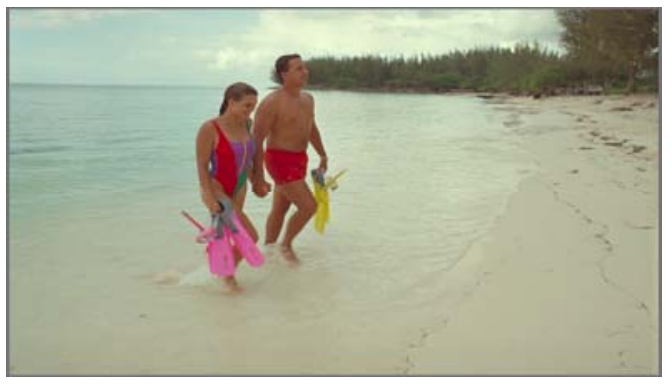

b) Beach

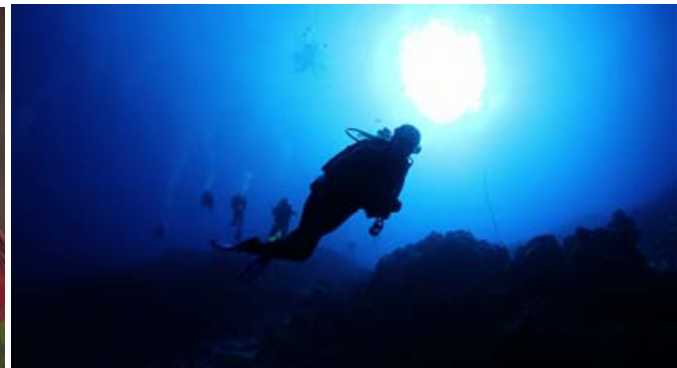

d) LA Diver

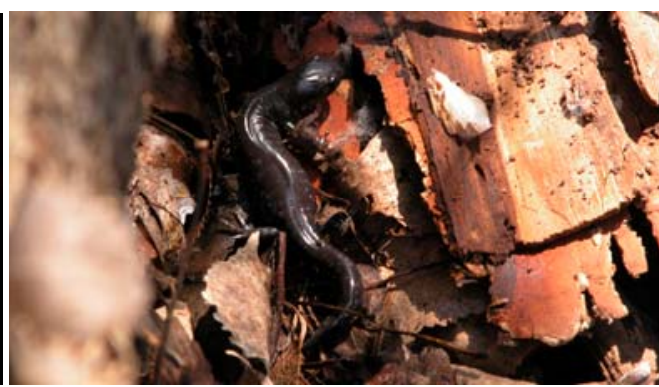

f) Lizard

Figure 8. Original six test images used in the experiment.

Table 1. Properties of different brightness preservation schemes summarized.

\begin{tabular}{|c|c|c|}
\hline Scheme & Advantages & Disadvantages \\
\hline a) Hard clipping without hue preservation & Simplicity; the highest overall brightness & $\begin{array}{l}\text { Strong clipping saturation (loss of details); } \\
\text { hue distortion }\end{array}$ \\
\hline b) Hard clipping with hue preservation & $\begin{array}{l}\text { Simplicity; high overall brightness; preserves } \\
\text { hue }\end{array}$ & Strong clipping saturation; loss of brightness \\
\hline c) Soft clipping against local maximum & $\begin{array}{l}\text { Good preservation of the relative tones and } \\
\text { details locally }\end{array}$ & $\begin{array}{l}\text { Complexity; relative uniformity between } \\
\text { dim and bright backlight areas reduced }\end{array}$ \\
\hline d) Soft clipping against global maximum & $\begin{array}{l}\text { Simplicity; avoids clipping saturation in bright } \\
\text { areas; preserves relative hue globally }\end{array}$ & $\begin{array}{l}\text { Does not preserve the overall brightness as } \\
\text { well as hard clipping }\end{array}$ \\
\hline e) Linear enhancement against local maximum & Excellent preservation of relative tones locally & $\begin{array}{l}\text { Complexity; relative uniformity between } \\
\text { dim and bright backlight areas reduced }\end{array}$ \\
\hline f) No compensation & $\begin{array}{l}\text { Simplicity; good preservation of hue in the } \\
\text { regions of bright backlight }\end{array}$ & $\begin{array}{l}\text { Dark backlight regions strongly dimmed; } \\
\text { backlight gradient visible in smooth surfaces }\end{array}$ \\
\hline
\end{tabular}




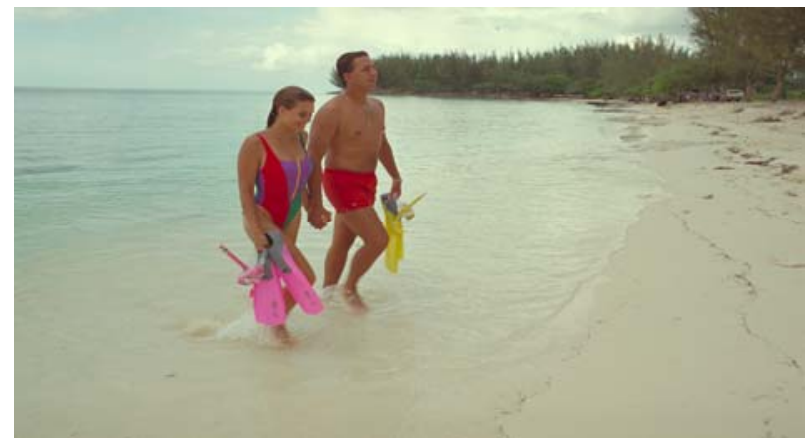

a) Original image

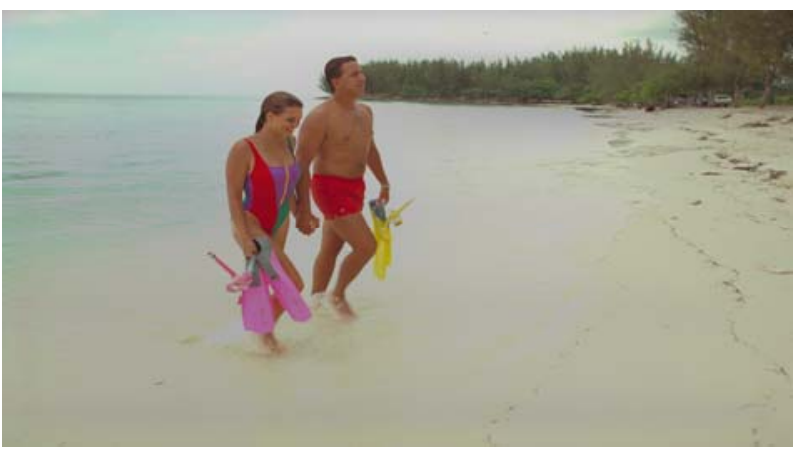

c) Hard clipping, no hue preservation

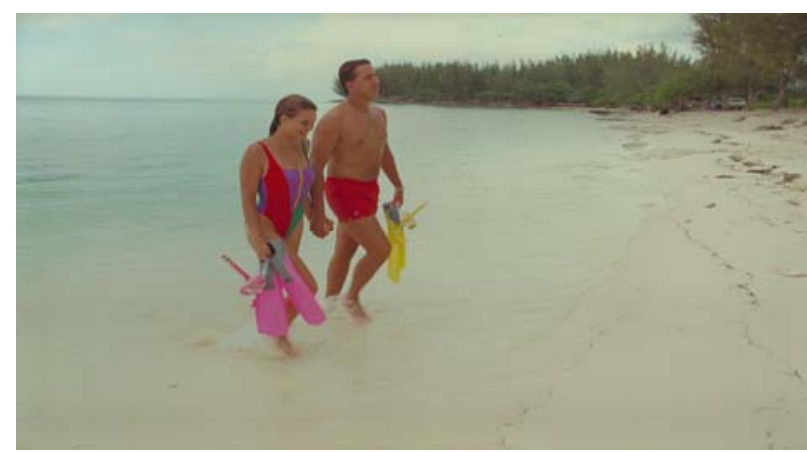

e) Soft clipping against local maximum

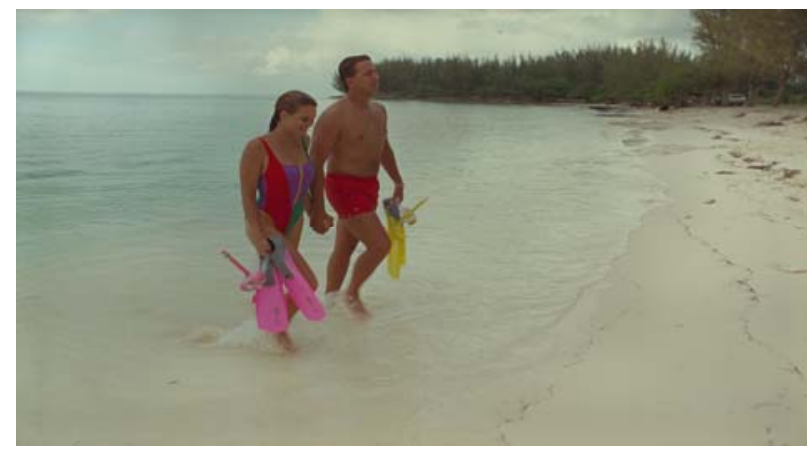

g) Linear enhancement against local maximum

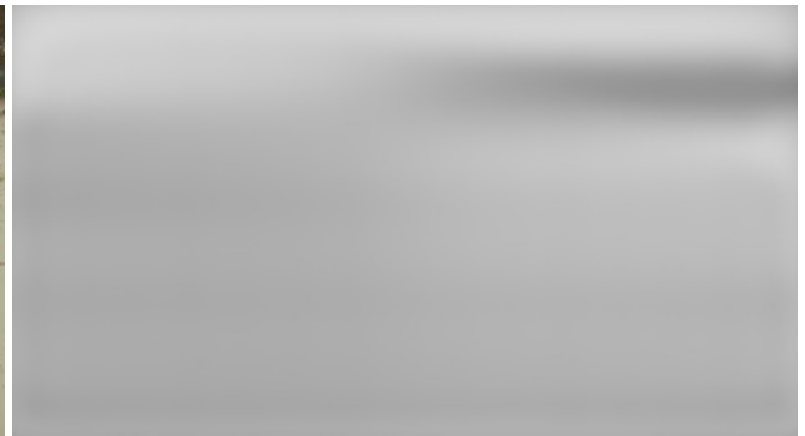

b) Backlight (from the backlight model)

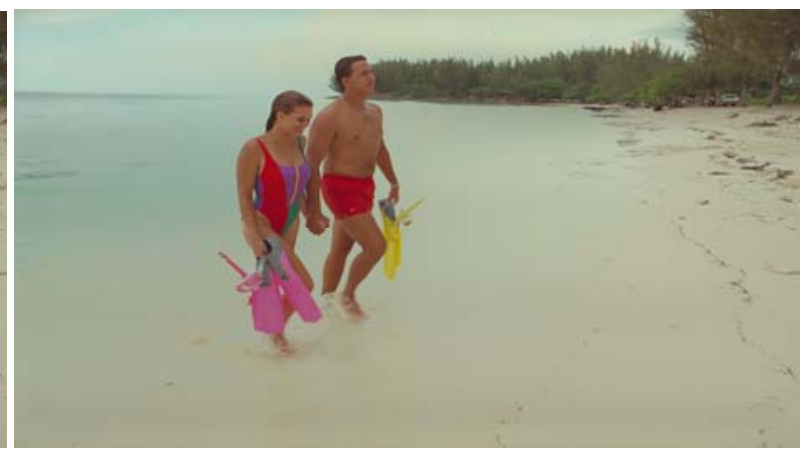

d) Hard clipping with hue preservation

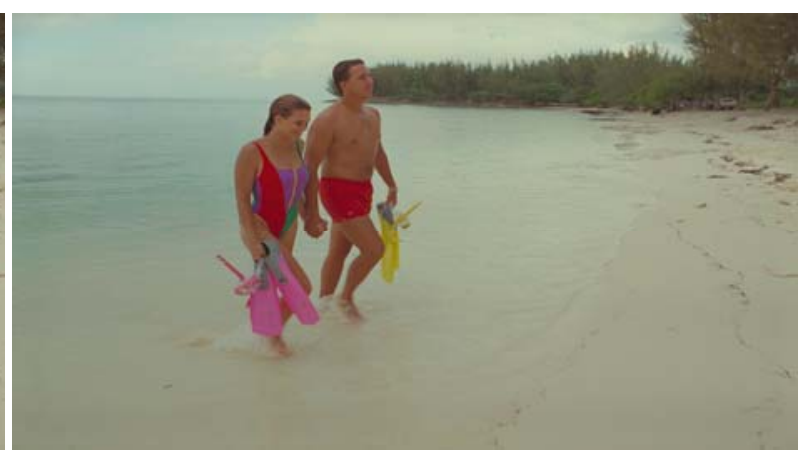

f) Soft clipping against global maximum

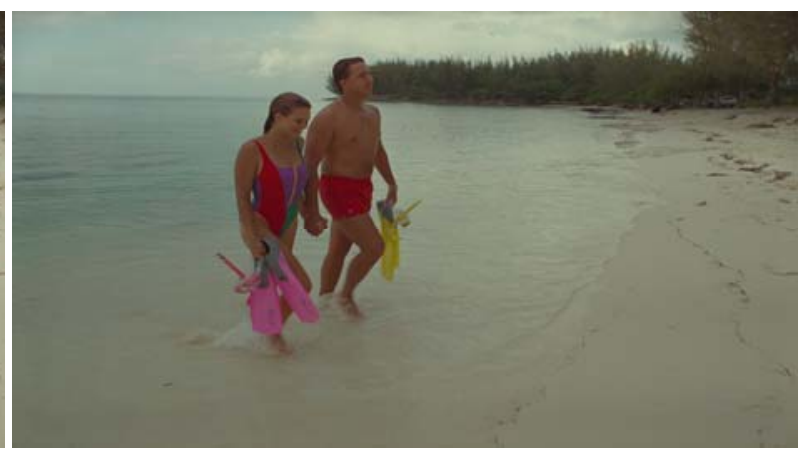

h) No compensation

Figure 9. Visual comparison of different brightness preservation schemes on Beach image. 
In total, 17 test subjects participated in the experiment (11 male, 6 female, age between 19 and 32). All test subjects self-reported vision that is normal or corrected to normal, with no impairments in color vision. None of the subjects reported significant experience in image processing. Tests were run in a laboratory room with lights switched off. A high dynamic range display from SIM2 with 2202 individually controllable backlight elements was used as a test display. Distance to the display was three times the height of the display, and the UI display was located approximately midway between the test display and the test subject, positioned so that it does not block the view to the test display. First two images were used for training only, and they were excluded from the analysis. To compensate the possible impact of viewing order on the results, the actual test images were shown in a pseudorandom order. In addition, the initial order of different versions of the images in each test case was randomized.

\subsection{Results and discussion}

The average and median rankings from the experiment are summarized in Table 2. Results have relatively large deviation of opinions from content to content, but in general, linear enhancement against local maximum gives the best average ranking for most images. A notable exception is LA Diver, showing the highest preference for direct hard clipping. Also for LA Volcano, direct hard clipping and local linear enhancement show almost equal average preference. Both LA Diver and LA Volcano are high contrast images with clearly distinguished dark and bright regions, and the results clearly indicate that preservation of peak intensity is more important in high contrast images than low contrast images. In bright images with lower contrast, preservation of local uniformity (i.e. preservation of the relative intensities of nearby pixels in respect with the original image) seems more important than preservation of peak intensity.

Figure 10 shows the boxplots illustrating the confidence intervals of subjective ratings for each test image. Exotic

Table 2. Mean and median (in parenthesis) ranking of tested brightness preservation schemes for each image (the best mean and median subjective ranking for each content is bolded).

\begin{tabular}{ccccccc}
\hline \hline Image & $\begin{array}{c}\text { Hard clipping } \\
\text { direct }\end{array}$ & $\begin{array}{c}\text { Hard clipping w. } \\
\text { hue preservation }\end{array}$ & $\begin{array}{c}\text { Soft clipping } \\
\text { (local max) }\end{array}$ & $\begin{array}{c}\text { Soft clipping } \\
\text { (global max) }\end{array}$ & $\begin{array}{c}\text { Local linear } \\
\text { enhancement }\end{array}$ & $\begin{array}{c}\text { No } \\
\text { compensation }\end{array}$ \\
\hline Exotic Flower & $3.88(4)$ & $3.82(4)$ & $3.06(3)$ & $\mathbf{2 . 9 4 ( 3 )}$ & $\mathbf{2 . 9 4 ( 3 )}$ & $4.35(5)$ \\
Beach & $5.47(6)$ & $5.06(5)$ & $3.35(4)$ & $3.06(3)$ & $\mathbf{1 . 5 3 ( 1 )}$ & $2.53(2)$ \\
Parrot & $5.52(6)$ & $5.18(5)$ & $3.18(4)$ & $3.00(3)$ & $\mathbf{1 . 4 1 ( 1 )}$ & $2.71(2)$ \\
LA Diver & $\mathbf{1 . 6 5 ( 1 )}$ & $2.65(2)$ & $2.59(3)$ & $4.53(5)$ & $3.71(4)$ & $5.88(6)$ \\
LA Volcano & $2.82(2)$ & $3.76(4)$ & $2.88(3)$ & $3.94(4)$ & $\mathbf{2 . 6 4 ( 2 )}$ & $4.94(6)$ \\
Lizard & $5.00(6)$ & $3.99(3)$ & $3.41(4)$ & $3.29(3)$ & $\mathbf{2 . 7 6 ( 2 )}$ & $3.06(\mathbf{2})$ \\
\hline All & $4.06(5)$ & $3.99(5)$ & $3.08(3)$ & $3.46(3)$ & $\mathbf{2 . 5 0 ( 2 )}$ & $3.91(4)$ \\
\hline \hline
\end{tabular}

Flower, LA Volcano and Lizard show relatively large deviation of subjective rankings. On the other hand, rankings for Beach, Parrot and LA Diver are substantially less scattered. Kruskal-Wallis tests reject the null hypothesis (random distribution at probability 5\% or higher) for all test images, except Exotic Flower. Beach and Parrot have relatively similar distributions with local linear enhancement giving clearly the best rank. For other images, the results are more ambiguous, possibly because the visible differences between brightness preservation methods are less obvious.

In general, direct hard clipping has only slightly worse overall ranking than hard clipping with hue preservation, which seems counterintuitive. In fact, for dark images, such as LA Diver and LA Volcano, direct hard clipping is clearly preferred to hard clipping with hue preservation. A possible explanation is that a small shift from blue towards cyan (Diver) or orange towards yellow (Volcano) is considered aesthetically insignificant or even a pleasant change. However, given that the primary purpose of brightness preservation is to maintain fidelity with respect to the original image rather than perform aesthetic enhancement, we do not recommend omitting hue preservation in hard clipping, even though it produces subjectively preferred outcome in some cases.

Our results indicate that for bright images with low contrast, it is more essential to avoid saturation and preserve the relative uniformity of pixels than to maintain high brightness level. In these cases, hard clipping should definitely not be 
Exotic Flower

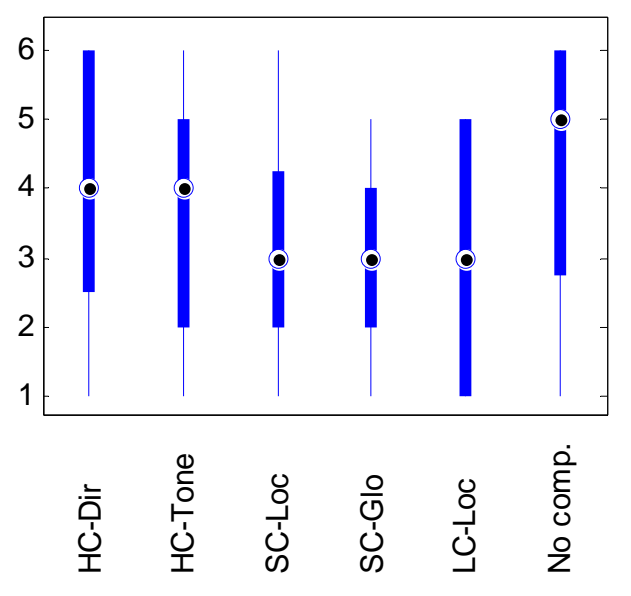

Parrot

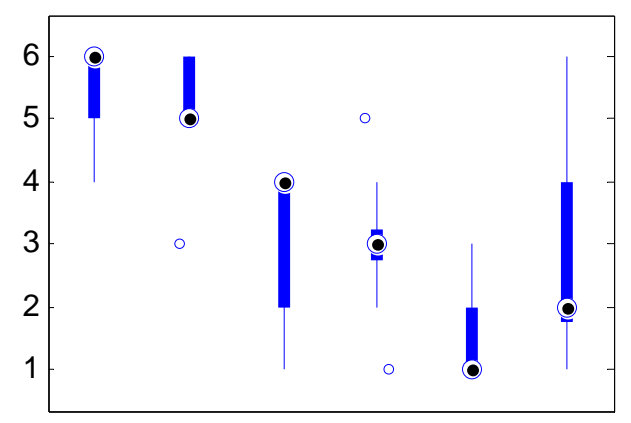

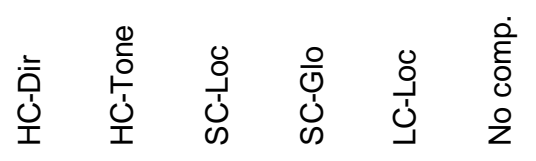

LA-Volcano

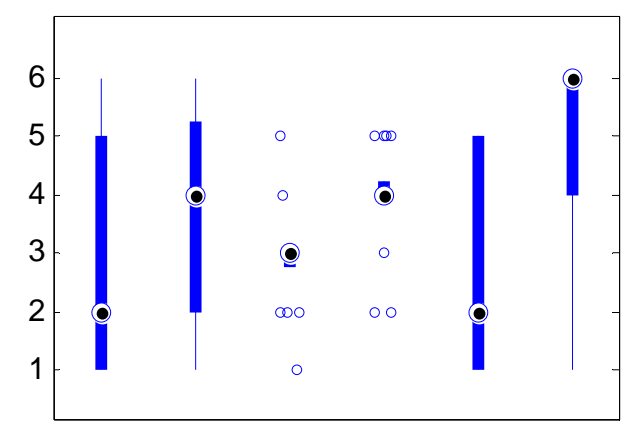

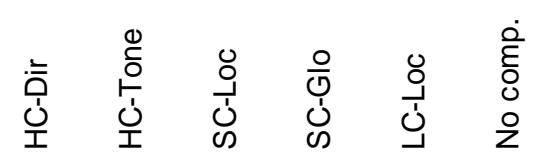

Beach

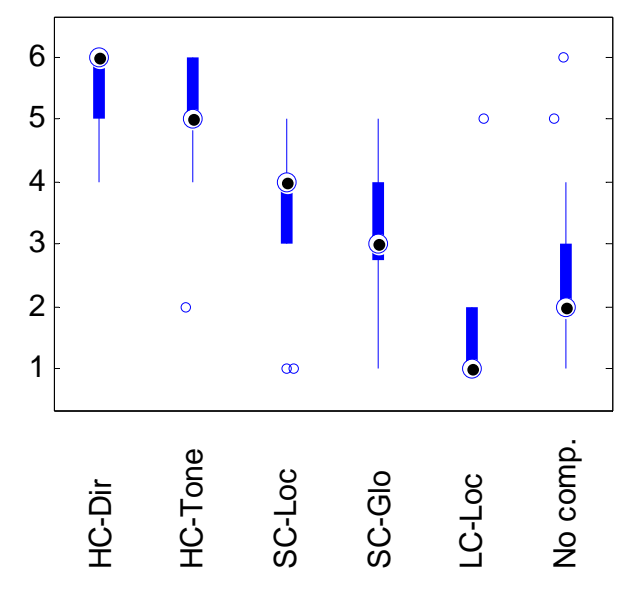

LA-Diver

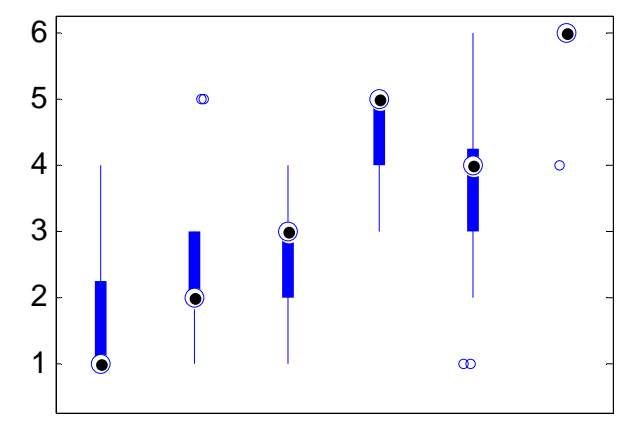

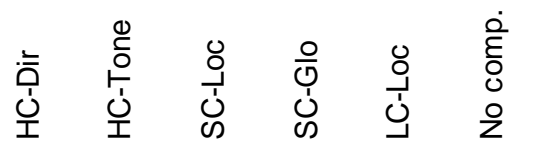

Figure 10. Boxplots depicting distributions of subjective rankings (thick bar shows the $50 \%$ range). HC-Dir: hard clipping direct, HC-Tone: hard clipping with hue preservation, SC-LoC: soft clipping (local max), SC-Glo: soft clipping (global max), LC-LoC: linear enhancement in respect to local maximum, No comp: no compensation. 
used. The most preferred alternative is local linear enhancement, followed by soft clipping with locally defined clipping curve. The results suggest that Human Visual System (HVS) is more sensitive to local uniformity than global uniformity; in other words, it is more essential to preserve the relative brightness differences between pixels located close to each other than between pixels located in different regions further away from each other. This can be explained by the fact that human eye can only focus sharply on a small area at a time, and LCDs using local backlight dimming are usually large in size.

The disadvantage of locally defined clipping curves is that it requires an algorithm to define a local maximum for each pixel. This increases the complexity of the implementation and computational requirements. If local enhancement is not feasible, globally defined soft clipping is a reasonable compromise between hard clipping and no compensation. Hard clipping works better for high contrast images (Diver and Volcano), whereas no compensation is preferred to hard clipping for some bright images with relatively uniform overall brightness and therefore also backlight (Beach and Parrot). For images with intermediate brightness and contrast (Exotic Flower and Lizard), soft clipping outperforms both hard clipping and no compensation.

Since there are some conceptual similarities between tone mapping and brightness preservation, interesting perspectives could be opened by comparing our study and subjective studies evaluating Tone Mapping Operators (TMOs). TMOs are traditionally classified into global and local methods. This distinction is comparable to the distinction between globally and locally defined brightness preservation in our study: global methods use similar (possibly content adaptive) mapping for all pixels, whereas local methods use locally adaptive mapping. Somewhat surprisingly, subjective studies tend to show higher preference for global TMOs $[8,18]$. This highlights the conceptual differences between tone mapping and brightness preservation (as explained in Section 1); however, tone mapping has been studied more intensively than brightness preservation, and future advances in brightness preservation techniques could result in different conclusions.

\section{CONCLUSIONS}

In this study, we have performed a subjective image quality experiment to compare different brightness preservation strategies for local backlight dimming displays. In general, brightness preservation must compromise between high overall brightness and uniformity with respect to the target image. We have observed that in most cases, locally adapted linear enhancement produces the most preferred visual outcome. This approach preserves the local uniformity well, but at the cost of reduced global uniformity and overall brightness. However, for high contrast images, higher overall preference can be achieved by using hard clipping, which preserves brightness better than the other methods, but at the cost of clipping saturation. Based on those observations, we have outlined guidelines for selection of the preferred pixel enhancement scheme in different situations.

\section{REFERENCES}

[1] Albrecht, M., Karrenbauer, A., Jung, T., and Xu, C., "Sorted Sector Covering Combined with Image Condensation: An Efficient Method for Local Dimming of Direct-Lit and Edge-Lit LCDs," IEICE Trans. Electr. E93-C(11), 1556-1563 (2010).

[2] Shu, X., Wu, X., and Forchhammer, S., "Optimal Local Dimming for LC Image Formation with Controllable Backlighting,” IEEE Trans. Image Process. 22(1), 166-173 (2013).

[3] Zhang, X.-B., Wang, R., Dong, D., Han, J.-H., and Wu, H.-X., "Dynamic Backlight Adaptation based on the Details of Image for Liquid Crystal Displays,” IEEE/OSA J. Disp. Tech., 8(2), 108-111 (2012).

[4] Mantel, C., Burini, N., Korhonen, J., Nadernejad, E., and Forchhammer, S., "Quality Assessment of Images Displayed on LCD Screen with Local Backlight Dimming," Proc. QoMEX, Klagenfurt, Austria (2013).

[5] Kerofsky, L., and Daly, S., "Brightness Preservation for LCD Backlight Dimming," J. of the SID, 14/12, pp. 1111-1118, 2006.H. Nam, "A Color Compensation Algorithm to Avoid Color Distortion in Active Dimming Liquid Crystal Displays,” IEEE Trans. Consumer Electr. 56(4), 2569-2576 (2010). 
[6] Cho, S.I., Kang, S.-J., Kim, Y.H. "Image Quality-Aware Backlight Dimming with Color and Detail Enhancement Techniques,” IEEE/OSA J. Display Tech. 9(2), 112-121 (2013).

[7] Tsai, P.-S., Liang, C.-K., Huang, T.-H., and Chen, H., "Image Enhancement for Backlight-Scaled TFT-LCD Displays,” IEEE Trans. Circ. and Syst. for Video Tech. 19(4), 574-583 (2009).

[8] Čadík, M., Wimmer, M., Neumann, L., and Artusi, A., "Evaluation of HDR Tone Mapping Methods Using Essential Perceptual Attributes," Computers \& Graphics 32(3), 330-349 (2008).

[9] Song, M., Tao, D., Chen, C., Bu, J., Luo, J., and Zhang, C., "Probabilistic Exposure Fusion,” IEEE Trans. Image Proc. 21(1), 341-357 (2012).

[10] Yeganeh, H., and Wang, Z., “Objective Quality Assessment of Tone-Mapped Images,” IEEE Trans. Image Proc. 22(2), 657-667 (2013).

[11] Korhonen, J., Burini, N., Forchhammer, S., and Pedersen, J. M., "Modeling LCD Displays with Local Backlight Dimming for Image Quality Assessment,” Proc. SPIE7866 (2011).

[12] Burini, N., Nadernejad, E., Korhonen, J., Forchhammer, S., and Wu, X., "Modeling Power-constrained Optimal Backlight Dimming for Color Displays,” IEEE/OSA J. Display Tech. 9(8), 656-665, (2013).

[13] Korhonen, J., Mantel, C., Burini, N., and Forchhammer, S., "Modeling the Color Image and Video Quality on Liquid Crystal Displays with Backlight Dimming," Proc. VCIP, Kuching, Malaysia (2013).

[14] ITU-R, “ITU-R Recommendation BT.500-12: Methodology for the Subjective Assessment of the Quality of Television Pictures” (2009).

[15] Franzen, R., "Kodak True Color Image Suite," <http://r0k.us/graphics/kodak/>

[16] Olmos, A., and Kingdom, F.A.A., "A Biologically Inspired Algorithm for the Recovery of Shading and Reflectance Images," Perception 33(12), 1463-1473 (2004).

[17] NTIA/ITS, “Consumer Digital Video Library," <http://www.cdvl.org/>

[18] Eilertsen, G., Wanat, R. Mantiuk, R., and Unger, J., "Evaluation of Tone Mapping Operators for HDRVideo," Computer Graphics Forum 32(7), 275-284 (2013). 